Positive Illusions in ADHD Girls - 1

RUNNING HEAD: POSITIVE ILLUSIONS IN ADHD GIRLS

Positive Illusions of Social Competence in Girls with and without ADHD

Jeneva Ohan \& Charlotte Johnston

AUTHOR PREPUBLICATION DRAFT 


\begin{abstract}
We compared social self-competence ratings in 9 to 12 year old girls with $(n=42)$ versus without ( $n=40)$ ADHD, relative to ratings of the girls' social competence made by mothers, teachers, and blind raters duringa social laboratory task. Relative to scores from mothers, teachers, and the lab-task, girls with ADHD over-estimated their competence significantly more than control girls. Over-estimates were greater for girls with ADHD who also had heightened oppositional-defiant symptoms, or lower depressive symptoms. Over-estimates were positively related to a socially desirable reporting bias for girls with ADHD, but not for control girls, suggesting that girls with ADHD attempt to present themselves in an unduly positive, self-protective light. For girls with ADHD, over-estimates also were positively related to maladjustment and negatively related to adjustment. However, for girls without ADHD, over-estimates were positively related to adjustment. Overall, over-estimates of competence function differently in girls with and without ADHD.
\end{abstract}




\title{
POSITIVE ILLUSIONS OF SOCIAL COMPETENCE IN GIRLS WITH AND WITHOUT
}

\author{
ADHD \\ Theory on the development of children's self-concept states that children with \\ successful experiences tend to develop a solid and healthy sense of self-competence, whereas \\ children with repeated experiences of failure tend to develop a low sense of self-competence \\ (Harter, 1981). Despite the intuitive logic of this hypothesis, this explanation does not hold \\ for all children. Children with aggressive interactions (David \&Kistner, 2000), learning \\ disabilities (Heath \& Glen, 2005), and attention-deficit/hyperactivity disorder (ADHD; e.g., \\ Hoza et al., 2004; Owens \&Hoza, 2003) all report positive self-competence, despite \\ consistently encountering failure. Much of the research on children's positive illusions of \\ their competence has focused on children with ADHD, a prevalent disorder characterized by \\ marked levels of inattention and/or hyperactivity-impulsivity (American Psychiatric \\ Association (APA), 2000). The difficulties faced by children with ADHD are serious, wide- \\ ranging, and enduring. For example, they suffer from low academic achievement, parent- \\ child conflict, poor relations with teachers, and significant peer difficulties (Barkley, 2006). \\ This multitude of impairments makes the positive self-perceptions of children with ADHD all \\ the more puzzling (see Owens, Goldfine, Evangelista, Hoza\& Kaiser, 2007 for a review). \\ A child's over-estimation of his/her own competence relative to his/her actual \\ competence is described as a "positive illusory bias (PIB)" (Hoza, Pelham, Dobbs, Owens, \& \\ Pillow, 2002). The PIB is operationalized as the difference between children's reports of their \\ self-competence and scores from a criterion measure, such as teacher reports of the child's \\ competence (Hoza et al., 2002). Studies utilizing this discrepancy score approach have \\ consistently found that children with ADHD over-estimate their social, academic, and \\ behavioral self-competence relative to reports from parents and teachers, and that these over-
}


estimates are significantly larger than those of children without ADHD (e.g., Evangelista, Owens, Golden, \& Pelham, 2008; Hoza et al., 2002, 2004).

Studies on the PIB in children with ADHD have shown clear differences in the magnitude of the PIB depending on comorbidity. Specifically, children with ADHD and comorbid conduct-oppositional problems most strongly over-estimate their competence relative to children with ADHD in the absence of ODD (Hoza et al., 2004; Hoza et al., 2002). On the other hand,children with ADHD and depressive symptoms are less likely to overestimate their self-competence relative to those with ADHD but not depression (Hoza et al., 2004).However, research on ADHD subtype differences is less clear, with only one study examining subtypes. Owens and Hoza (2003) found that children with children with only the inattentive symptoms of ADHDwere less likely to over-estimate their academic competence than were children with both the inattentive and hyperactive-impulsive symptoms of ADHD. However, they did not simultaneously consider comorbidity issues, and thus the results may reflect ODD comorbidity in the children with hyperactive-impulsive symptoms, and/or depression in the children with inattentive-only symptoms (Owens et al., 2007).

Studies that have defined the PIB as a discrepancy between child reports and criterion measures have relied heavily on parent and/or teacher ratings as the criteria. However, research on informant discrepancies in reports of child psychopathology shows that no single assessor provides a "gold standard" as each offers a different perspective on the child's behavior across contexts (e.g., De Los Reyes\&Kazdin, 2004).As such, it is possible that the discrepancy between child and adult ratings reflects some combination of child overestimation and biases or perspective differences in teacher and parent ratings. Using both adult ratings and laboratory or performance based measures of the child's competence as criteria, Owens and Hoza (2003) examined the academic self-competence ratings of children with ADHD symptoms and control children relative to standardized reading and math tests, 
and to teacher reports of the children's academic competence. Compared to the control group, children with ADHD symptoms rated their academic competence significantly higher than teachers (Cohen's $\mathrm{d}=.83$ ), but only moderately higher than their actual math test scores $($ Cohen's $d=.37$ ) and not significantly higher than their reading scores. Thus, this study provides evidence that the degree of children's over-estimations vary across criteria measures, and suggests that including a performance-based measure against which children's self-reports also can be judged adds additional information about the PIB.

Although previous studies have found evidence for the PIB in both boys and girls with ADHD (e.g., Evangelista et al., 2008; Hoza et al., 2004), the number of girls in these studies remains small.Further study of the PIB in girls with ADHD is needed, especially in the social domain. Compared to boys, girls have a stronger relational orientation (e.g., Maccoby, 1990), have stronger links between relational difficulties and psychological maladjustment (e.g., Crick \& Nelson, 2002), and are more reactive to negative interpersonal events (e.g., Rudolph, 2002). These factorsmay increase the potential for a PIB in estimating social competence to be particularly damaging as it would reduce the ability of girls with ADHD to accurately self-assess and self-correct their behavior in this important realm of functioning. Like boys with ADHD, girls with ADHD display a variety of damaging social interactions, including high levels of confrontational relational (social) aggression, overt (physical) aggression, awkwardness, and poor social skills (Ohan\& Johnston, 2007; Zalecki\&Hinshaw, 2004). In addition, girls with ADHD appear to be more impaired socially than boys with ADHD. For example, girls with ADHD are more disliked by their peers than are boys with ADHD (Gaub\& Carlson, 1997), presumably because the tightly-woven friendship groups of girls are less forgiving of these deviant behaviors than are the looser, more physical friendship groups of boys (Ohan\& Johnston, 2007; Zalecki\&Hinshaw, 2004). 
If, as the evidence indicates, children with ADHD over-estimate their competence, one important question is: what is the purpose of the PIB?Of the handful of existing explanations (see Owens et al., 2007, for a review), the most strongly supported is the "selfprotective hypothesis" (Diener\&Milich, 1997). This hypothesis suggests that children with ADHD over-estimate their skills in order to mask their true sense of inadequate ability and to present a positive facade or 'bravado' to others. Not only is this explanation consistent with findings that children with ADHD are most likely to over-estimate their competence in their area of greatest weakness (Hoza et al. 2004, 2002), but it also has stood up well to direct testing. For example, Diener and Milich (1997) found that when boys diagnosed using DSMIII-Rcriteria for ADHD received positive feedback about their task social interaction with a peer (i.e., had their positive image confirmed), they lowered their estimates of their social performance. Other tests of the self-protective hypothesis have provided similar results for boys with ADHD regardless of subtype (Ohan\& Johnston, 2002).However, the selfprotective hypothesis has not yet been tested in girls with ADHD.

Although the PIB may function by providing children with ADHD a way to preserve a positive image, researchers have recently questioned whether or not the PIB is ultimately helpful (e.g., Owens et al., 2007). In their milestone review of optimism and mental health, Taylor and Brown (1988) argued that, "the mentally healthy person appears to have the enviable capacity to distort reality in a direction that enhances self-esteem, maintains beliefs in personal efficacy, and promotes an optimistic view of the future" (p. 205). They conceptualized positive illusions as being particularly evident and adaptive under adverse circumstances, and we argue that the continual experiences of failure that are common to children with ADHD would fall within this description. Other theorists disagree with Taylor and Brown'sformulation. For example, Colvin, Block, and Funder (1995) view accurate selfjudgment as the cornerstone of mental health, and thus positive illusions as maladaptive. A 
middle-ground between these two approaches is that moderately positive illusions may be helpful to overall well-being, with only extreme illusions having maladaptive consequences (e.g., Baumeister, 1989; Paulhus, 1998). Although links between the PIB and psychosocial functioning in children have been demonstrated in community and mixed clinical samples (e.g., Gresham, Lane, MacMillan, Bocian, \& Ward, 2000; Kistner, David, \&Repper, 2007), this has not been examined inchildren with ADHD. Such research in ADHD samples is especially pressing given that acknowledging one's challenges may predict psychosocial treatment success. For example, Mikami et al. (2010) recently reported that the PIB was negatively associated with psychosocial treatment outcome for children with ADHD.

\section{Current Study Hypotheses}

The first purpose of this study was to extend our knowledge of the PIB in girls with versus without ADHD by examiningnot only the discrepancies between children's selfreports of their social competence and reports of their mothers and teachers, but also relative to their performance on alaboratory-based performance measure. Second, consistent with previous research (e.g., Hoza et al., 2002; 2004), we also examined the role of comorbidoppositional-defiant and depressive symptoms in the PIB of children with ADHD.The third purpose of our study was to extend previous tests of the self-protective hypothesis by examining the association between girls' over-estimates of their social competence and their socially-desirable (self-enhancing) responding. If the self-protective hypothesis is correct, then strong correlations between the PIB and social desirability should be seen for children with ADHD given their motivation to present an unrealistically positive image to the world (Deiner\&Milich, 1997), but these relations should be significantly smaller for control girls who experience less failure and presumablyhave a lesser need to self-protect. Finally, based on arguments that the level of PIB may moderate its relation to psychological 
well-being, we examined the link between the PIB and psychosocial well-being in both groups of girls.

\section{Methods}

\section{Participants}

Forty-two girls with ADHD and 40 girls without ADHD aged 9 to 12 years and their mothers participated. Participants were recruited through newspaper, school, and community advertisements, and notices in ADHD support group newsletters, a hospital's ADHD clinic, and physician offices. Demographic characteristics are presented in Table 1. Of the 42 girls with ADHD, 33 were taking stimulants. Mothers, teachers, and girls were instructedto completemeasures describing the child's behavior off medication to the best of their ability, and girls were taken off medication 24 hours prior to participating (mean withdrawal $=28 \mathrm{~h}$ ).

In order to be included in the ADHD group, girls had to meet two inclusion criteria. First, they needed to be diagnosed by a qualified professional (14 made by primary physicians, 12 by psychologists, 11 by psychiatrists, and 5 by pediatricians). Second, girls needed to currently meet DSM-IV ADHD criteria (APA, 2004), based on motherand teacher ratings over the past 6 months on the ADHD Rating Scale-IV (ADHD-RS-IV; DuPaul, Power, Anastopoulos, \& Reid, 1998). The ADHD-RS-IV includes the 18 DSM-IV ADHD symptoms on a 4-point scale from 0 (never or rarely) to 3 (very often), and ratings above the mid-point (i.e., pretty often or very often) are counted as a present symptom. There is very good evidence for reliability and validity of the DSM-RS-IV (DuPaul et al., 1998) and for the use of such ratings in the diagnostic process (Pelham, Fabiano, \&Massetti, 2005). In the present study, internal consistencies for the total score were .85 and .82 for mothers' and teachers' ratings of the ADHD group and .84 and .81 for mothers' and teachers ratings of the control group. Motherratings indicating the presence of at least six symptoms of either inattention or hyperactivity-impulsivity were required. Based on mothers' ratings, 18 of the 
girls with ADHD met symptom criteria for the inattentive subtype and 24 met the combined subtype. Teachers also ratedgirlsin the ADHD group on the DSM-RS-IV. All girls in the ADHD group met criteria according to teachers' symptom ratings except for three, all of whom were being treated with stimulants during the school day.These girls were rated as having at least four inattention symptoms or four hyperactive-impulsive symptoms, and thus were considered appropriate for the ADHD group. Finally, mothers and teachers rated children on the Children's Impairment Rating Scale (Fabiano et al., 2006; see psychometric properties in measures section below). Scores in the ADHD group overwhelmingly indicated impairment in girls with vs without ADHD based on mothers' $t(1,83)=13.44, p<.001$, and teachers' $, t(1,81)=13.91, p<.001$, ratings.

In order to be included in the control group, mothers had to rate their daughters as having less than six symptoms on both subscales of the ADHD-RS-IV (all girls had 3 or fewer symptoms on each subscale). Exclusionary criteria for both groups included pervasive developmental disorders (e.g., autism) and mental retardation, assessed by mother report and supported by the child's teacher.

\section{Measures}

\section{Measures of Social Competence}

Matson Evaluation of Social Skills for Youngsters (MESSY; Matson, Rotatori, \&Helsel, 1983). The MESSY, a comprehensive self- and teacher-report scale of social competence for 4- to 18- year olds, was our measure of parent, teacher, and child-reported social competence. As has been done by others (e.g., Bell-Dolan \& Allan, 1998), we created a parent-report version parallel to the teacher-report version as these items are easy for parents to rate as well. Respondents rate the child, or the child rates himself/herself, on 26 items describing socially competent peer interactions (e.g., "This child does (I do) nice things for people who are nice to him or her (me)," "This child joins (I join) in games with other 
children”) on a 5-point Likert scale. This scale shows expected developmental effects, and has been used in normative and clinical samples, with internal consistency and test-retest reliability ranging from adequate to excellent (e.g., Matson et al., 1983; Matson, Macklin, \&Helsel, 1985). In the present study, internal consistencies were good to excellent (ADHD group, mother reports: .76, teacher reports: .78, child reports: .91; Control group, mother reports: .80 , teacher reports: .78, child reports: .86). Further supporting the use of the MESSY with parents of girls in our sample, the correlation between mother and teacher reports was $.43, p<.01$ in the ADHD and $.40, p<.01$, in the control group.

Laboratory social competence measure: the “Girl's Club!'(Ohan\& Johnston, 2007). The "Girl's Club!" game is a computerized board game created to assess social interactions in girls in a controlled laboratory setting. Social interactions assessed during this game have been shown to have excellent reliability (e.g., high internal consistencies) and validity (e.g., overlap with mother and teacher reports) in girls with and without ADHD (for a detailed description, see Ohan\& Johnston, 2007). Briefly, a female research assistant (RA) told each girl that she was playing on-line with two other girls of the same age and grade who lived in the same city, and that they would all meet after the game. In reality, all of the moves and comments from the co-players were simulated and pre-programmed and the same for every girl. Girls were fully debriefed at the end of the game.

The game began with the RA reading the instructions, after which each girl was asked if she wanted to play, and to either press a "Yes" button to start the game or the "No thanks!" button to decline (no girl took this option). Girls were then presented with a computerized board game and moved around the board by dice rolls. To reduce the possibility that the RA would influence the girls' responses, the RA sat behind the computer and read a magazine. All moves and dice rolls during the game were pre-determined. Throughout the game, there were five "Chat Centers" that allowed the girl to send a message to her co-players, and these 
messages were coded into prosocial, aggressive, and awkward categories. As a criterion measure of social competence, the prosocial (i.e., socially skilled, competent, and friendly) comments sent at these centres were used. Examples of these comments include, "you guys $r$ FUN to play with and COOL," "you guys r the best!!," and "I really like playing with you guys." Coders also rated the intensity of each message from 1 (least prosocial) to 5 (extremely prosocial). For example, the message "hi" received a score of 1, whereas “Congratulations for being in the lead!! I like Computer pets, too!" received a 5. To keep this measure on the same metric as the other social competence measures (the MESSY uses a 5point scale), and to have an indication of the average social competence shown throughout the game, the mean intensity score was calculated.

The first author and two students served as coders, with $50 \%$ of responses independently coded by two individuals. All coders had access only to transcripts with no identifying information and were blind to group status (ADHD vs Control). Discrepancies were dealt with at meetings, and the consensus was the final score. The kappa coefficient for the prosocial category was very good (.77). Intraclasscorrelations determined the agreement amongst raters on intensity scores, and agreement was excellent (.92).

\section{Measures of Comorbid Symptoms and Sub-group Definitions}

The Oppositional Defiant Disorder Rating Scale (ODDRS; Hommersen, Murray, Ohan, \& Johnston, 2006). We used mothers' ratings of ODDsymptoms as a measure of oppositional problems. This scale contains the DSM-IV ODD criteria, rated on a 4-point rating scale, as well as questions about the chronicity and impairment. The ODDRS has satisfactory internal consistency, inter-rater and test-retest reliability, and convergent validity (Hommersen et al., 2006). Internal consistencies in this study were 90 for the ADHD and .84 for the control group. Using ratings above the mid-point of the 0 to 3 scaleas indicating symptom presence, along with endorsement of chronicity and impairment criteria, 22 of the 
42 girls in the ADHD group met DSM-IV ODD criteria of at least four ODD symptoms (the ADHD/ODD subgroup), and the other 20 girls with ADHD did not (ADHD/noODD; $n=20$ ). As expected, there was an overlap between ADHD subtype and ODD symptoms such that 20 of 22 girls with ADHD-Combined type (ADHD-C) also met ODD group criteria, butonly 2 of 20 girls with ADHD-Inattentive type (ADHD-IA)met ODD group criteria.

Children's Depression Inventory(CDI; Kovacs, 1992). The CDI is a widely accepted child self-report measure of depression. Children rate 27 items on a 3-point Likert scale, with greater scores indicating greater depression. For this study, the total CDI score was used. The CDI has good internal consistency, test-retest reliability, and validity (Kovacs et al., 1992). The internal consistencies in the current study were very good (ADHD, .90; control, .84). Consistent with previous studies (e.g., Hoza et al., 2002, 2004), we used a T-score cutoff of 60 to define our subgroups of girls with ADHD and depressive symptoms (ADHD/Dep; $n=$ 18) and girls with ADHD without depressive symptoms (ADHD/noDep, $n=24$ ). As expected, there was an overlap between ADHD subtype and depressionsuch that 12 of 20 girls with ADHD-IA also met depression group criteria, and only 6 of 22 girls with ADHD-Combined typemet depression group criteria.

Measure of Socially Desirably Responding

Revised Children's Manifest Anxiety Scale-Lie Scale (RCMAS-Lie; Reynolds \& Richmond, 1978). The Lie Scale is a widely-used measure of socially desirable or defensive responding in elementary-school children (e.g., Dadds, Perrin, \& Yule, 1998). It contains nine yes/no items describing socially desirable but unrealistically positive characteristics (e.g., "I never get angry," "I tell the truth every single time"). The psychometric properties of the Lie scale are generally excellent, with strong test-retest reliability, internal consistencies and kappa reliability estimates, content validity as indexed by overlap with the MMPI-Lie 
scale, and evidence for convergent and divergent validity (e.g., Reynolds \& Richmond, 1978). The internal consistency for girls with ADHD was .88 and for controls was .86 . Measures of Psychosocial Functioning

Number of friends and play-dates. Mothers and teachers estimated the number of girls' friends. Their estimates were highly correlated (ADHD group, .72; control group, .71), and the mean was therefore calculated. Mothers reported the number of friends who had come to play with their daughter during the past week, and the number of times that their daughter had gone to a friend's house to play during the past week. These estimates were similarly highly correlated (ADHD group, .70; control group, .76) and were added to estimate the number of play-dates per week. The total number of friends and number of play-dates shared a small-to-moderate correlation, (ADHD group, $r(41)=.37, p<.03$; control group, $r(41)=$ $.41, p<.02)$, suggesting that these constructs were best kept separate for analyses.

Children's Impairment Rating Scale (CIRS; Fabiano et al., 2006). The CIRS contains six items that reflect areas of children's functioning (peers, teacher relations, classroom behavior, self-esteem, academic difficulties, and global impairment). Each item is rated from 0 (no problem, definitely does not need treatment or special services) to 5 (extreme problem, definitely needs treatment or special services). The final rating asks an inverted question, that is, to what extent the child has overall strengths (none to definite strengths). The CIRS has good test-retest reliability and concurrent and discriminant validity (Fabiano et al., 2006). For the sake of parsimony, we report links with mothers' ratings only (results with teachers' ratings were very similar). The strengths item was used as a measure of adjustment, and the impairment items were summed to reflect general impairment (internal consistency of .80 in the ADHD group, and .92 in the control group).

Revised Children's Manifest Anxiety Scale-Anxiety Scale (RCMAS-Anxiety; Reynolds \& Richmond, 1978).This extensively-used anxiety scale for elementary-school 
childrencontains 28 yes/no items. There is a considerable evidence for the reliability and validity of the RCMAS-Anxiety scale (e.g., Reynolds \& Richmond, 1978). Internal consistencies in this study were .82 for the ADHD group and .90 for the control group.

Children's Social Behavior Scale-Parent Form (CSBS-P; Crick, 1996). The CSBS-P is a parallel form of a teacher report measure of overt and relational aggression. The subscales reported here include overt aggression (4 items) and relational aggression ( 7 items). For teachers' ratings, these scales have shown high internal consistency (.94), and high interrater reliability between peers' and teachers' ratings of relational aggression (.63) and overt aggression (.54) in girls (Crick, 1996). For the present study, internal consistencies of mothers' reports were very good to excellent (ranging from a low of .80 for control girls' overt aggression to a high of .94 for relational aggression in the control group).

\section{Procedure}

This study was approved by our university's ethics board. Mothers who contacted the laboratory were informed of the procedures,completed the ADHD DSM-IV-RS and were mailed a copy of the consent form and teacher questionnaires. In a few cases, teacher-report was not informative or possible (e.g., the child had recently moved to a new school, it was the start of the school year) and another appropriate adult rater who was not a relative was identified. For both groups, over $90 \%$ of reporters were teachers (for the ADHD group, 2 were leaders of girl-guide (also known as girl scout) groups and 2 were after-school care providers; for the Control group, 2 were after-school care providers). All informants knew the girl for at least 2 months (mean, 22.32 months, $\mathrm{SD}=5.43$ months).

The teacher returned his/her completed questionnaire to the mother in a signed and sealed envelope, which the mother brought to the lab. Upon arrival in the lab, the mother gave signed informed consent and completed her measures, while the research assistant and daughter proceeded to the lab. Once in the lab, the daughter was asked what she knew about 
the computer game. If there was any reason to believe that the girl's mother had informed her as to the purposes of the game or that the game players were computer simulations, that girl's data was excluded (this happened for 2 girls of an original 42 girls in the control group).

After the game, the girl was debriefed and told that her co-players were computer simulations. The RA explained the purpose of the game (i.e., to see how girls interact with each other, and if their interaction is different if they have ADHD) as well as why deception was necessary to achieve these purposes (i.e., because if they knew that the other co-players were not real then they might have acted differently, and we would not know how girls interact with each other). Girls typically showed surprise that their co-players were not real, but none appeared upset that this was the case, and most commented that they would have acted differently if they had known their co-players were not real so they thought it best that they did not know before they played the game. After this, every girl was thanked and given a T-shirt in appreciation, and the mother was given a $\$ 20$ honorarium.

\section{Results}

\section{Demographic Group Differences}

Girls in the control versus ADHD groups were compared using $t$-tests for number of siblings, mother age, years parents married, age, grade, and socio-economic status. Chi square tests examined group differences in the frequency of ethnic groups, married versus single mothers, and proportion of employed mothers and fathers. No comparisons were significant ( $p s>.11)$.

\section{Calculation of Discrepancy Scores}

As in previous studies in this area, our measure of the positive illusory bias is the discrepancy between children's self-reports and other measures of social competence (mother ratings, teacher ratings, and observer coding of the lab task). Positive discrepancies represent the child's over-estimates and negative scores represent under-estimates of skills (Hoza et al., 
2004). Although difference or discrepancy scores are not without statistical limitations, we believe they are a conceptually appropriate way to operationalize the PIB. Problems with discrepancy scores arise if the component scores show ceiling or floor effects (which was not a problem with our measures; see Table 3) or if they differ in their variances (De Los Reyes \&Kazdin, 2004). To correct for differences in variances, it is recommended that standardized discrepancies by used (De Los Reyes \&Kazdin, 2004; Owens et al., 2007). We did so by first calculating z-scores for the child self-reports and for each criterion measure, and then taking the difference between these $\mathrm{z}$ scores.

Despite their psychometric superiority, one disadvantage of the z-score discrepancies is that the scores can no longer be interpreted in their original units. Take, for example, comparable measures with means and standard deviations of 18 and 3 for child reports and 10 and 2 for teacher reports. A hypothetical girl with a self-rated score of 17 and a teacher-rated score of 13 would be over-estimating her abilities relative to her teacher's view (raw score difference: $17-13=+4$ ). However, if these scores are first transformed into z-scores, the discrepancy is negative ( $\mathrm{z}$-score difference: $-.33-1.50=-1.83$ ) indicating that, relative to other girls, this girl places herself below average while the teacher places her above average relative to other girls. For our purposes, we used the standardized discrepancy scores for analyses given their psychometric superiority (Owens et al., 2007), but we also note raw or unstandardizedscore discrepancies alongside the standardized score discrepancies in tables in order to provide a full picture of under-or over-estimates of competence.

In total, we had three discrepancy scores for reports of social skills: child-mother, child-teacher, and child-lab task. These three scores were correlated for the girls within the ADHD group, $(r(41)=.66, .44$, and .49 for the child-mother with child-teacher, child-mother with child-lab-task, and child-teacher with child-lab task, respectively) and within the control group $(r(41)=.61, .42$, and .52 for the same pairings; all $p s<.01)$. 


\section{Group and Sub-group Differences in Discrepancy Scores}

First, we examine group differences in the discrepancy scores (child-mother, childteacher, and child-lab task) using multivariate analyses of variance (MANOVA) models. We then compare ADHD subgroups formed based on comorbidities (ADHD plus and minus ODD and depressive symptoms) to each other and to the control group. To control for experiment-wise error, a Bonferroni correction was used for these three MANOVAs, resulting in alphas of .016 for significance. To further examine overall differences that were significant, univariate analyses of variance (ANOVA) models and Least Significant Difference (LSD) post-hoc tests were conducted, and significance for these follow-up tests were held at the conventional $p<.05$ level.

\section{ADHD versus Control Group differences}

For the MANOVA model that compared the ADHD and control groups with the three discrepancy scores as dependent measures, there was a significant main effect of group, $F(1$, $81)=4.95, p=.005$, partial $\eta^{2}=.15$. Univariate ANOVAs were significant for the discrepancy between child and mother reports of social competence, $F(1,81)=6.68, p=$ .002 , partial $\eta^{2}=.08$, child and teacher reports, $F(1,81)=15.00, p<.001$, partial $\eta^{2}=.15$, and child report and performance on the lab task, $F(1,79)=5.23, p=.009$, partial $\eta^{2}=.06$. Girls in the ADHD group significantly over-estimated their social competence relative to girls in the control group. For discrepancy means and standard deviations, see Table 3.

\section{ODD Symptom Sub-group Differences}

The MANOVA model comparing girls with ADHD with ODD symptoms (ADHD/ODD), girls with ADHD but without ODD symptoms (ADHD/noODD), and girls in the control group also was significant, $F(6,170)=3.78, p=.002$, partial $\eta^{2}=.11$. Univariate ANOVAs were significant for the discrepancy between child and mother reports of social competence, $F(1,81)=10.41, p<.001$, partial $\eta^{2}=.14$, child and teacher reports, $F(1,81)=$ 
$12.04, p<.001$, partial $\eta^{2}=.21$, and child report and performance on the lab task, $F(1,79)=$ $6.48, p=.002$, partial $\eta^{2}=.14$. LSD post-hoc tests conducted on the child-mother discrepancy scores indicated greater over-estimates for girls with ADHD/ODD versus the ADHD/noODD girls and control girls. Post-hoc tests for the child-teacher and child-lab task discrepancy scores were all significant, such that girls with ADHD/ODD significantly overestimated their social competence relative to the ADHD/noODD girls, who in-turn significantly over-estimated their social competence relative to girls in the control group. For means and standard deviations, see Table 3.

Note on ADHD subtype. As in many ADHD samples, ADHD subtype and ODD were highly related in our sample and this overlap makes it difficult to discern the extent to which differences between girls with and without comorbid ODD are due to ODD versus hyperactive-impulsive behavior. We therefore redid the MANOVA for ODD comorbidity using ADHD hyperactive-impulsive symptoms as a covariate. The results less strong but remained significant, $F(6,170)=2.72, p=.021$, partial $\eta^{2}=.10$, suggesting that ODD comorbidity influences the PIB above and beyond hyperactive-impulsive symptoms.

\section{Depressive Symptom Sub-group Differences}

The MANOVA comparing girls with ADHD and depressive symptoms (ADHD/Dep), girls with ADHD but without depression (ADHD/noDep), and girls in the control group was significant, $F(6,170)=2.74, p<.010$, partial $\eta^{2}=.09$. Univariate ANOVAs were significant for the discrepancy between child and mother reports, $F(1,83)=3.65, p<.029$, partial $\eta^{2}=$ .08 , child and teacher reports, $F(1,83)=7.62, p<.001$, partial $\eta^{2}=.15$, and child report and performance on the lab task, $F(1,79)=3.25, p=.031$, partial $\eta^{2}=.07$. LSD post-hoc tests conducted on the child-mother, child-teacher, and the child-lab task discrepancy scores all indicated greater over-estimates for girls in the ADHD/noDep group versus those with ADHD/Depand those in the control group. For descriptive information, see Table 3. 
Note on ADHD subtype. As with ODD and hyperactive-impulsive symptoms, we redid the above MANOVA for depression groupcomorbidity using ADHD inattentive symptoms as a covariate. The results were less strong but still marginally significant, $F$ (6, $170)=2.08, p<.097$, partial $\eta^{2}=.06$, suggesting that depression comorbidity influences the PIB above and beyond inattentive symptoms.

\section{Relations Between Discrepancy Scores and Social Desirability}

We examined the correlations between scores on the RCMAS-Lie scale and each of the discrepancy scores (child-mother, child-teacher, and child-lab task), separately by group. Given that RCMAS Lie and Anxiety scores shared small but significant correlations for both groups, $r(41)=.27, p=.041$ for the ADHD group, and $r(39)=.25, p=.044$ for the control group, we controlled for RCMAS Anxiety scores. Confirming our hypothesis, for girls with ADHD, RCMAS-Lie scores were significantly and positive correlated with the child-mother discrepancy, $r(41)=.47, p=.006$; the child-teacher discrepancy, $r(41)=.39, p=.009 ;$ and the child-lab task discrepancy, $r(41)=.29, p=.038$. None of these correlations were significant in the control group, $r \mathrm{~s}-.02$ to -.12. To test if the size of the RCMAS-Lie and discrepancy score correlations differed between the ADHD and control groups, Fisher Z transformations were tested. All correlations significantly differed in magnitude $(p s<.05)$.

\section{Relations Between Discrepancy Scores and Measures of Psychosocial Functioning}

For the ADHD group, eight of the nine correlations between girls' discrepancy scores and measures of positive functioning were significant and negative. Greater over-estimates of social competence were related to poorer functioning as measured by number of friends, play-dates with friends, and overall strengths. However, for girls in the control group, six of the nine correlations were significant, but in the positive direction, indicating that overestimates of social competence in this group were related to better functioning. For the measures reflecting problems in psychosocial functioning, for girls in the ADHD group, 
discrepancy scores were unrelated to anxiety symptoms but were generally significantly and positively related to both overt and relational aggression. The measure of overall impairment was significantly related to all discrepancy measures for girls in the ADHD group. For control girls, discrepancy scores were negatively correlated with anxiety symptoms, but none of the correlations between discrepancy scores and aggression or overall impairment were significant. See Table 4 for correlations.

To address the concern that these relations are dependent on the links between psychosocial functioning and social competence itself (and not the child vs other-report discrepancy), we conducted partial correlations between psychosocial functioning and the discrepancy scores, controlling for the criterion measure (parent-report, teacher-report, and lab-task score). Although the overall strength of the relations was decreased, the overall pattern of correlations remained largely the same. According to Fisher Z tests used to test the significance of the magnitude of the relations before and after partial correlations, only one correlation (that between the overall rating of the child's strengths and the child-mother social competence discrepancy) was significantly changed. See Table 4.

\section{Discussion}

Due to their symptoms and related difficulties in academics and social interactions, children with ADHD consistently encounter failure. Despite concerns that these failure experiences will be detrimental to the self-esteem of these children, research has found that children with ADHD (typically boys) often hold more positive perceptions of their academic, social, and behavioral competence than appears warranted (Owens et al., 2007). Our study extended this literature by showing that girls with ADHD also over-estimated their social competence when their reports were considered relative to mothers and teachers, as well as a laboratory observation of social competence. Thus, not only do these girls evidence social competence deficits, but they fail to report or acknowledge these deficits to a greater degree 
than do non-problem girls. The range of scores on the social competence measures and the use of standardized discrepancy scores argue against these over-estimates being a statistical artifact of the lower competence scores of girls with ADHD. Furthermore, these overestimates were most notable for girls with ADHD and comorbidODDsymptoms, and least marked for girls with ADHD and depressive symptoms. These findings firmly demonstrate that many PIB findings previously reported only for boys with ADHD also are characteristic of girls with the disorder.

In addition to replicating previous findings, our results also offer an important extension of our understanding of the PIB both in relation to its possible function and its adaptive value. When we examined the relations between girls' over-estimates and a socially desirable response bias as well as indices of psychosocial well-being, very different patterns of relations emerged for girls in the ADHD versus control groups. For girls with ADHD, over-estimates were positively related to socially desirable responding, externalizing problems, and global impairment, and negatively linked to psychosocial adjustment. However, for girls in the control group, over-estimates were not related to socially desirable responding, and tended to be negatively related to internalizing pathology, and positively related to psychosocial adjustment. In sum, it appears that for girls with ADHD, consistent with the self-protective hypothesis, the PIB is linked to a tendency to present one's self in an unrealistically positive light, and in the context of the higher levels of PIB in these girls compared to controls, their over-estimates are associated with poor psychosocial adjustment. However, for control girls, over-estimates do not appear to reflect socially desirable responding, and are actually associated with better psychosocial adjustment.

\section{Over-estimates of Social Competence}

The unstandardized discrepancy scores indicated that both groups of girls in this study over-estimated their social competence relative to their mothers, their teachers, and a 
laboratory task. That girls in the control group tended to rate their competence somewhat higher than the judgments made by mothers, teachers, and blind observers is consistent with the self-enhancing bias often seen in normal samples (e.g., Alicke\&Govorun, 2005; Gaertner, Sedikides, \& Chang, 2008). This also is in keeping with theory on the development of childhood self-perceptions and competence, which conceptualizes children as moving from gross over-estimates of their abilities in the preschool years to more reasonable levels by midto late-childhood (e.g., Harter, 1981). However, girls with ADHD over-estimated their competence significantly more than girls in the control group. We speculate that the selfperceptions of girls with ADHD may follow a delayed development path relative to control girls, or they may alternatively follow a different path, such as remaining high throughout development. Research that tests these pathways is greatly encouraged.

That girls with ADHD over-estimated their social competence more than controls relative to mother and teacher report was expected based on previous findings (e.g., Hoza et al., 2002, 2004). However, given the possibility that adult reports of social competence in children with ADHD may be affected by different perspectives or biases (e.g., Jackson \& King, 2004), one of our purposes was to examine whether or not this discrepancy would still exist if girls' reports were considered relative to an in-vivo observation (in our case, a social lab task using blind raters). Our results indicated that, compared to control girls, girls with ADHD significantly over-estimated their social competence relative to the lab task criterion, indicating that the PIB phenomenon is not only found in comparison to adult ratings. Still, the effect size for the lab task comparison was somewhat smaller than for comparisons with mother and teacher ratings. These results echo previous findings (Owens \&Hoza, 2003) and may be explained by multiple factors. It is possible that mother and teacher reports are negatively influenced by difficult interactions with the child. Alternatively, because mothers and teachers are able to observe children's social interactions across contexts, ages, and 
genders, their more negative ratings may reflect the reality of the child's poor social competence, which is less accurately captured by a brief lab task.

Similar to previous studies, we also found that the magnitude of the PIB differed based on comorbiditybetween ADHD and ODD or depression (e.g., Hoza et al., 2002, 2004). In our study, girls with ADHD and ODD symptoms had the highest levels of positive illusions, even after controlling for hyperactive-impulsive symptoms. This suggests that the defiant, aggressive, and hostile interpersonal style of girls with ODD, at least when in the presence of $\mathrm{ADHD}$, is also typified by an unduly positive intrapersonal style. This positive self-image may engender even greater levels of negativity toward authority figures who, through making remarks or engaging in arguments, may unintentionally challenge the child's self-image. Future research on whetheror not positive illusions contribute to or are a consequence of ODD is greatly encouraged. We also found thatgirls who had ADHD with lower levels of depressive symptoms had more positive illusions than girls who had ADHD with high depressive symptoms or girls in the control group. That girls with ADHD and depression hold self-perceptions that are largely consistent with girls in the control group suggests that co-occurring depression may counteract the positive illusions typically associated with ADHD, leading to average self-perceptions rather than the negative views of self typically associated with depression.

\section{Self-Protective Hypothesis}

Our study also offered a test of the self-protective hypothesis, which argues that the PIB allows children with ADHD to present a positive front to the world and thus maintain self-esteem (Deiner\&Milich, 1997). This hypothesis predicts a positive relationship between positive presentation bias and the PIB in children with ADHD. Correlations between socially desirable responding and the PIB for the ADHD group were strong and significant. Notably, 
these correlations were consistently smaller in size and non-significant for girls in the control than ADHD group, presumably because they are not as motivated to present a positive image.

One question that lingers is whether or not children with ADHD consciously manipulate or truly believe the positive front that they portray? In social psychology, it is well accepted that social desirability has two components, one that reflects a genuine belief in one's overly positive reports (called self-deception) and another a tendency to distort one's image as positive for the purpose of an audience (called impression management or otherdeception) (e.g., Paulhus, 1984). The social desirability scale used in the present investigation (the RCMAS-Lie scale) contains mostly impression-management items (e.g., "I am always polite," "I tell the truth every time"). Given the solid correlations between the Lie scale and the PIB measures in girls with ADHD, we can infer that at least some of their positive illusions represent a conscious defense against feelings of inadequacy. However, this does not rule out the possibility that the PIB is also related to self-deception. Unfortunately, the lack of an existing measure of self-deception for children makes this question difficult to address.

\section{Relations between the PIB and Psychosocial Well-being}

We also examined links between the PIB and psychosocial functioning, an area that has been debated but unstudied among children with ADHD. For girls with ADHD, overlypositive reports of social competence were related to lower levels of psychosocial well-being, with the exception of anxiety. However, for girls in the control group, over-estimates of social competence were generally related to greater psychosocial well-being, and unrelated to aggression or overall impairment. This pattern was largely echoed when a more stringent test was applied, using the criterion raw score (mother-report, teacher-report, or lab-task rating) as a covariate, implying that these relations are not dependent on the relation between psychosocial functioning and social competence itself. 
Views on how adaptive positive illusions are for an individual's mental health have been hotly debated for decades (e.g., Colvin et al., 1995; Taylor \& Brown, 1988). Overall, our findings indicate that relations between positive illusions of social competence and psychosocial functioning may differ by group, presumably because of group differences in the degree of positive illusion. Taylor and Brown's (1988) argument that positive illusions are adaptive may best characterize the findings for girls in the control group, who consistently showed mild to moderate over-estimates of their social competence that were related to positive adjustment. However, Colvin and colleagues' (1995) view of positive illusions as maladaptive may most appropriately describe the girls with ADHD, whose large over-estimates of their social competence were consistently related to worse psychosocial adjustment. In sum, experts who have argued for a middle-ground approach - that is, that mild to moderate illusions may be helpful to overall well-being, while extreme illusions have maladaptive consequences -may be most correct (e.g., Baumeister, 1989; Paulhus, 1998).

\section{Clinical Implications}

Some experts (e.g., Owens et al., 2007) have expressed concern that the inflated selfviews of children with ADHD are likely to result in the child lacking motivation to challenge him/herself to develop necessary skills, as the child does not perceive (or admit to) weaknesses. Thus, the PIB may be an obstacle to instigating independent self-improvement in areas that are needed most, and indeed, there is evidence that the PIB is associated with poor treatment outcome (Mikami et al.,2010). This may be especially true for children with ADHD plus ODD, as their negative and hostile attitude towards others may be worsened or maintained by an overly positive self-view. Research on the role of the PIB in children with ODD is encouraged, as directly addressing positive self-views in therapy (such as part of a CBT-based intervention) may boost the effectiveness of treatment. 
Disentangling the proportion of positive illusions that are due to self-deception (or lack of awareness) versus impression management has implications for treatment. Children who are unaware of their limitations are likely to require different intervention strategies than children who are aware of, but defensive about, their short-comings (Hoza et al., 2002). On the one hand, if the PIB is related to self-deception and children with ADHD are largely unaware of their deficits, then strategies such as teaching more accurate self-evaluation and skills may be appropriate (Hoza et al., 2002). On the other hand, to the extent that children with ADHD are engaging in impression management in order to present themselves in an unrealistically positive light, as we have found evidence for here, therapy will need to address this defensive coping style. Gresham and colleagues (1998) have argued that providing children who have positive illusions of their competence with further encouragement will only fuel future problems. We believe that strategies that explicitly deal with defensiveness and resistance, such as those that form the basis of motivational interviewing or motivational enhancement therapy, combined with cognitive-behavioral strategies to teach more adaptive coping strategies may prove suitable.

\section{Limitations}

Although this study advances our understanding of the PIB, particularly in girls with ADHD, there are limitations. First, we believe the focus on an exclusive female sample was justified by the lack of research in this population and the importance of social competence and interpersonal functioning for females. However, the lack of boys precludes a direct analysis of gender differences in the PIB. Although previous studies that have included both boys and girls have found no differences in the size of the PIB, future tests of gender differences across multiple ages and domains of functioning, and using a variety of criterion measure are clearly needed. Second, the PIB is currently conceptualized and analyzed as the discrepancy between an informant's and a child's report of the same skill domain. However, 
this does not take into account that baseline functioning levels between the groups may be influencing the observed group differences.Future research in the area may benefit from identifying a priori a single measure as a control variable to be used in analyses of group differences. Ideally, such a measure would not be used in the formation of the PIB scores. As a related point, including discrepancies between self-reports and a variety of different criterion measures, such as peer-ratings, is an important avenue for understanding the extent and function of the PIB and for accounting for the limitations of the various criterion measures. Third, as already noted, the lack of a measure of self-deception is a limitation that future research will need to address in order to more fully inform our understanding of the basis of the PIB in children with ADHD and the development of appropriate treatments. Moreover, we were not able to meaningfully compare the PIB in ADHD subtypes due to a large degree of overlap between ADHD subtype and ODD symptoms (i.e., 20 of 22 girls with ADHD-Combined type also met ODDgroup criteria, and 18 of 20 girls with ADHD-IA did not have ODD). This overlap makes it difficult to discern the extent to which differences between girls with ADHD+ODD and ADHD-only are due to ODD versus hyperactiveimpulsive behavior. Future research using a 'pure' ADHD-C group, a combined ADHD-C and ODD group, and a 'pure' ODD group, will be needed to sort out this crucial question. Finally, our data is cross-sectional, limiting our ability to comment on the long-term helpfulness (or hindrance) of the PIB.

\section{Summary}

We found that girls with ADHD demonstrate a positive illusory bias in estimating their social competence, in comparison to both adult ratings and a lab-based measure of social competence. The PIB was highest in girls with ADHD and oppositional-conduct problems and lowest in those with depressive symptoms. Importantly, the positive illusory bias in girls with ADHD was significantly related to wanting to present oneself in positive 
light, and suggesting its maladaptive nature, was associated with various indices of maladjustment. These results point to the importance of understanding this aspect of the intrapersonal functioning of children with $\mathrm{ADHD}$, and call for attention to this bias in treatment planning.

\section{References}

Alicke, M. D., Govorun, O. (2005). The better-than-average effect. In M. D. Alicke, D. A. Dunning, \& J. I. Krueger (Eds.), The self in social judgment (pp. 85-106). Philadelphia, PA: Psychology Press.

American Psychiatric Association. (2000). Diagnostic and statistical manual of mental disorders (4th ed., text rev.). Washington, DC: Author.

Barkley, R. A. (2006). Attention-deficit hyperactivity disorder: A handbook for diagnosis and treatment ( $3^{\text {rd }}$ ed.). New York: Guilford.

Baumeister, R. F. (1989). The optimal margin of illusion. Journal of Social and Clinical Psychology, 8, 176-189.

Bell-Dolan, D. J., \& Allan, W. D. (1998). Assessing elementary school children's social skills: Evaluation of the parent version of the Matson evaluation of social skills with youngsters. Psychological Assessment, 10, 40-48.

Colvin, C. R., Block, J., \& Funder, D. C. (1995). Overly positive self-evaluations and personality: Negative implications for mental health. Journal of Personality and Social Psychology, 68, 1152-1162.

Crick, N. R. (1996). The role of overt aggression, relational aggression, and prosocial behavior in the prediction of children's future social adjustment. Child Development, 67, 2317-2327. 
Crick, N. R., \& Nelson, D. A. (2002). Victimization within peer relationships and freindships: Nobody told me there's be friends like this. Journal of Abnormal Child Psychology, 30, 599-607.

Dadds, M. R., Perrin, S., \& Yule, W. (1998). Social desirability and self-reported anxiety in children: An analysis of the RCMAS lie scale. Journal of Abnormal Child Psychology, 26, 311-317.

David, C. F., \& Kistner, J. A. (2000). Do positive self-perceptions have a "dark side"? Examination of the link between perceptual bias and aggression. Journal of Abnormal Child Psychology, 28, 327-337.

De Los Reyes, A., \& Kazdin, A. E. (2004). Measuring informant discrepancies in clinical child research. Psychological Assessment, 16, 330-334.

Diener, M. B., \& Milich, R. (1997). Effects of positive feedback on the social interactions of boys with attention deficit hyperactivity disorder: A test of the self-protective hypothesis. Journal of Clinical Child Psychology, 26, 256-265.

DuPaul, G. J., Power, T. J., Anastopoulos, A. D., \& Reid, R. (1998). ADHD Rating Scale-IV: Checklists, norms, andclinical interpretation. New York: Guilford.

Evangelista, N. M., Owens, J. S., Golden, C. M., \& Pelham, W. E., Jr. (2008). The positive illusory bias: Do inflated self-perceptions in children with ADHD generalize to perceptions of others? Journal of Abnormal Child Psychology, 36, 779-791.

Fabiano, G. A., Pelham, W. E., Jr., Waschbusch, D. A., Lahey, B. B., Onyango, A. N., Lopez-Williams, A., et al. (2006). A practical measure of impairment: Psychometric properties of the Impairment Rating Scale in samples of children with attention deficit hyperactivity disorder and two school-based samples. Journal of Clinical Child and Adolescent Psychology, 35, 369-385. 
Gaertner, L., Sedikides, C., \& Chang, K. (2008). On pancultural self-enhancement: Welladjusted Taiwanese self-enhance on personally valued traits. Journal of CrossCultural Psychology, 39, 463-477.

Gaub, M., \& Carlson, C.L. (1997).Gender differences in ADHD: A meta-analysis and critical review. Journal of the American Academy of Child and Adolescent Psychiatry, $36,1036-1045$.

Gresham, F. M., Lane, K. L., MacMillan, D. L., Bocian, K. M., \& Ward, S. L. (2000). Effects of positive and negative illusory biases: Comparisons across social and academic selfconcept domains. Journal of School Psychology, 38, 151-175.

Gresham, F. M., MacMillan, D. L., Bocian, K. M., Ward, S. L., \& Forness, S. R. (1998). Comorbidity of hyperactivity-impulsivity-inattention and conduct problems: Risk factors in social, affective, and academic domains. Journal of Abnormal Child Psychology, 26, 393-406.

Harter, S. (1981). A model of mastery motivation in children: Individual differences, developmental change. In W. A. Collins (Ed.), Aspects of the development of competence (pp. 215-225). Hillsdale, NJ: Erlbaum.

Heath, N. L., \& Glen, T. (2005). Positive illusory bias and the self-protective hypothesis in children with learning disabilities. Journal of Clinical Child and Adolescent Psychology, 34, 272-281.

Hollingshead, A. B. (1975). Five-factor index of social status. Department of Sociology, Yale University, Princeton, NJ.

Hommersen, P., Murray, C., Ohan, J. L., \& Johnston, C. (2006). Oppositional defiant disorder rating scale: Preliminary evidence of reliability and validity. Journal of Emotional and Behavioral Disorders, 14, 118-125. 
Hoza, B., Gerdes, A. C., Hinshaw, S. P., Arnold, L. E., Pelham, W. E., Molina, B. S. G., et al. (2004). Self-perceptions of competence in children with ADHD and comparison children. Journal of Consulting and Clinical Psychology, 72, 382-391.

Hoza, B., Pelham, W. E., Dobbs, J., Owens, J. S., \& Pillow, D. R. (2002). Do boys with attention-deficit/hyperactivity disorder have positive illusory self-concepts? Journal of Abnormal Psychology, 111, 268-278.

Jackson, D. A., \& King, A. R. (2004). Gender differences in the effects of oppositional behavior on teacher ratings of ADHD symptoms. Journal of Abnormal Child Psychology, 32, 215-224.

Kistner, J., David, C., \& Repper, K. (2007). Self-enhancement of peer acceptance: Implications for children's self-worth and interpersonal functioning. Social Development, 16, 24-44.

Kovacs, M. (1992). Children's Depression Inventory manual. North Tonawanda, NY: MultiHealth Systems.

Maccoby, E. E. (1990). Gender and relationships: A developmental account. American Psychologist, 45, 513-520.

Matson, J. L., Macklin, G. F., \& Helsel, W. J. (1985). Psychometric properties of the Matson Evaluation of Social Skills with Youngsters (MESSY) with emotional problems and self concept in deaf children. Journal of Behavior Therapy and Experimental Psychiatry, 16, 117-123.

Matson, J. L., Rotatori, A. F., \& Helsel, W. J. (1983). Development of a rating-scale to measure social skills in children: The Matson Evaluation of Social Skills with Youngsters (MESSY). Behavior Research and Therapy, 21, 335-340.

Mikami, A.Y., Calhoun, C.D., \&Abikoff, H.B. (2010). The positive illusory bias and response to behavioral treatment among children with Attention-Deficit/ 
Hyperactivity Disorder.Journal of Clinical Child and Adolescent Psychology, 39, 373385.

Ohan, J. L., \& Johnston, C. (2002). Are the performance overestimates given by boys with ADHD self-protective? Journal of Clinical Child and Adolescent Psychology, 31, $230-241$.

Ohan, J. L., \& Johnston, C. (2007). What is the social impact of ADHD in girls? A multimethod assessment. Journal of Abnormal Child Psychology, 35, 239-250.

Owens, J. S., Goldfine, M. E., Evangelista, N. M., Hoza, B., \& Kaiser, N. M. (2007). A critical review of self-perceptions and the positive illusory bias in children with ADHD. Clinical Child and Family Psychology Review, 10, 335-351.

Owens, J. S., \& Hoza, B. (2003). The role of inattention and hyperactivity/impulsivity in the positive illusory bias. Journal of Consulting and Clinical Psychology, 71, 680-691.

Paulhus, D. L. (1984). Two-component models of socially desirable responding. Journal of Personality and Social Psychology, 46, 598-609.

Paulhus, D. L. (1998). Interpersonal and intrapsychic adaptiveness of trait self-enhancement: A mixed blessing? Journal of Personality and Social Psychology, 74, 1197-1208.

Pelham, W. E. Jr., Fabiano, G. A., \& Massetti, G. M. (2005). Evidence-based assessment of Attention Deficit Hyperactivity Disorder in children and adolescents.Journal of Clinical Child and Adolescent Psychology, 34, 449-476.

Reynolds, C. R., \& Richmond, B. O. (1978). What I think and feel: Revised measure of children's manifest anxiety. Journal of Abnormal Child Psychology, 6, 271-280.

Rudolph, K. (2002). Gender differences in emotional resposnes to interpersonal stress during adolescence. Journal of Adolescent Health, 30, 3-13.

Taylor, S. E., \& Brown, J. D. (1988). Illusion and well-being: A social psychological perspective on mental health. Psychological Bulletin, 103, 193-210. 
Positive Illusions in ADHD Girls - 33

Zalecki, C. A., \& Hinshaw, S. P. (2004). Overt and relational aggression in girls with attention deficit hyperactivity disorder. Journal of Clinical Child and Adolescent Psychology, 33, 125-137. 
Table 1

Demographic information (mean with standard deviation and range in parentheses) for girls with and without ADHD

ADHD

Socio-economic class ${ }^{\mathrm{a}}$

Number of DSM-IV Symptoms

Inattention

Hyperactive/Impulsive

Overall Impairment (CIRS) ${ }^{\mathrm{b}}$

Mother-rated

$3.97(1.40 ; 1-5)$

$3.61(1.22 ; 1-5)$

Teacher-rated
$126(1.22 ; 108-162)$

$41.40(6.06 ; 27-53)$

$44.63(7.85 ; 30-70)$

$89.90 \%$

$2.38 \%$

$2.38 \%$

$2.38 \%$

$2.38 \%$

$62.00 \%$

$97.40 \%$

$2.50(.67 ; 1-4)$

\section{Control}

$128(1.32 ; 108-173)$

$41.37(4.87 ; 31-53)$

$44.13(4.46 ; 37-53)$

$85.00 \%$

$7.50 \%$

$5.00 \%$

$2.50 \%$

$0 \%$

$68.70 \%$

$96.89 \%$

$2.38(1.01 ; 1-5)$

7.75 (1.23; 4-9)

$0.93(1.19 ; 1-4)$

$5.20(2.76 ; 3-9)$

$0.63(.92 ; 1-3)$ $0.24(0.65 ; 0-2)$

${ }^{a}$ Based on the 5-factor Hollingshead (1975) index. Higher scores indicate lower socio-

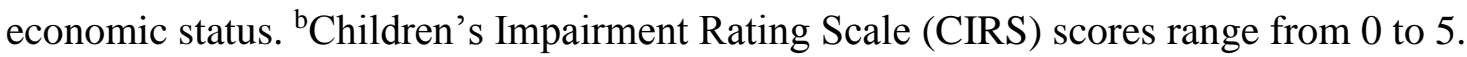


Table 2

Descriptive information about the completed measures (means with standard deviation and range in parentheses) for girls with and without $A D H D$

ADHD Control

Social Competence

$\begin{array}{lll}\text { Child-Reported (MESSY) } & 4.23(.63 ; 2.61-5.00) & 4.26(.40 ; 3.17-5.00) \\ \text { Mother-Reported (MESSY) } & 3.53(.54 ; 2.46-4.40) & 4.23(.44 ; 2.77-4.31) \\ \text { Teacher-Reported (MESSY) } & 3.47(.60 ; 2.35-3.98) & 4.17(.40 ; 2.65-4.01) \\ \text { Lab-Task } & 2.70(1.10 ; 0-4.33) & 3.45(1.07 ; 2.00-4.88)\end{array}$

ConcurrentSymptomatology

DSM-IV Oppositional-Defiant Symptoms

$\begin{array}{lll}\text { (mother-reported ODDRS) } & 4.19(1.45 ; 0-8) & 0.44(1.10 ; 0-3) \\ \text { Depression (CDI T-score) } & 58.78(7.28 ; 52-78) & 52.56(4.89 ; 46-58) \\ \text { Anxiety (RCMAS) } & 12.29(6.01 ; 3-25) & 7.36(4.76 ; 0-16) \\ \text { Relational Aggression (CSBS) } & 1.90(0.60 ; 1-3.40) & 1.20(.37 ; 1-2.10) \\ \text { Overt Aggression (CSBS) } & 1.48(.56 ; 1-3.08) & 0.56(.21 ; 1-1.85)\end{array}$

Social Desirability

\begin{tabular}{ll} 
Lie Scale (RCMAS) & $3.75(2.58 ; 0-8)$ \\
\hline
\end{tabular}

Note. MESSY $=$ the Matson Evaluation of Social Skills for Youngsters. CDI $=$ the

Children's Depression Inventory. RCMAS = the Revised Child Manifest Anxiety Scale.

ODDRS = Oppositional Defiant Disorder Rating Scale. CSBS $=$ Children's Social Behavior Scale. 
Table 3

Discrepancies in social competence ratings between child report and mother report, teacher report, and performance during the laboratory task by ADHD group and comorbidity

\begin{tabular}{|c|c|c|c|c|c|c|}
\hline \multirow[b]{4}{*}{ Groups } & \multicolumn{4}{|c|}{ Discrepancy Score } & & \\
\hline & \multicolumn{2}{|c|}{ Child-Mother } & \multicolumn{2}{|c|}{ Child-Teacher } & \multicolumn{2}{|c|}{ Child-Lab Task } \\
\hline & Unstand. & Standard & Unstand. & Standard & Unstand. & Standard \\
\hline & $\operatorname{Mean}(\mathrm{SD})$ & $\operatorname{Mean}(\mathrm{SD})$ & Mean(SD) & Mean(SD) & $\operatorname{Mean}(\mathrm{SD})$ & Mean(SD) \\
\hline \multicolumn{7}{|l|}{ ADHD Group } \\
\hline ADHD & $.91(.77)$ & $.38(.20)$ & $1.05(.57)$ & $.49(.17)$ & $.95(.83)$ & $.26(.17)$ \\
\hline Controls & $.61(.44)$ & $-.39(.21)$ & $.70(.49)$ & $-.45(.18)$ & $.64(.88)$ & $-.30(.18)$ \\
\hline \multicolumn{7}{|l|}{ ODD Symptoms } \\
\hline ADHD/ODD & $1.07(.87)$ & $.78(.26)$ & $1.23(.64)$ & $.82(.21)$ & $1.08(.88)$ & $.40(.22)$ \\
\hline ADHD noODD & $.76(.57)$ & $-.19(.31)$ & $.88(.38)$ & $.03(.25)$ & $.83(.86)$ & $.08(.26)$ \\
\hline Controls & $.61(.44)$ & $-.39(.21)$ & $.70(.49)$ & $-.45(.18)$ & $.64(.88)$ & $-.30(.18)$ \\
\hline \multicolumn{7}{|l|}{ Depression } \\
\hline ADHD/Dep & $.86(.67)$ & $.15(.29)$ & $.88(.63)$ & $.33(.32)$ & $.73(.96)$ & $-.45(.18)$ \\
\hline ADHD no Dep & $.98(.81)$ & $.46(.24)$ & $1.13(.55)$ & $.56(.20)$ & $.97(.49)$ & $-.05(31)$ \\
\hline Controls & $.61(.44)$ & $-.39(.21)$ & $.70(.49)$ & $-.45(.18)$ & $.64(.88)$ & $-.30(.18)$ \\
\hline
\end{tabular}

Note. Unstand. = Unstandardized; ADHD = attention-deficit/hyperactivity disorder; ODD = Oppositional Defiant Disorder; Dep = Elevated depressive symptoms. All contrasts significant at the $p<.05$ level. 
Table 4

Correlations between social competence discrepancy scores and psychosocial functioning

\section{Discrepancy Score}

Child-Mother Child-Teacher Child-Lab Task

\begin{tabular}{llll}
\hline Measures of Adjustment & \multicolumn{3}{c}{ ADHD group } \\
\cline { 2 - 4 } Number of friends & $-.32^{*}\left(-.22^{*}\right)$ & $-.36^{*}\left(-.24^{*}\right)$ & $-.24^{*}(-.14)$ \\
Play-dates with friends per week & $-.37^{* *}\left(-.26^{*}\right)$ & $-.25^{*}(-.15)$ & $-.10(-.02)$ \\
Overall rating of child's & $-.57^{* * *}\left(-.33^{* *}\right)$ & $-.33^{*}(-.21+)$ & $-.27^{*}(-.17)$ \\
strengths & & & \\
& & & \\
& & & \\
Number of friends & $.17(.08)$ & $.27^{*}(.12)$ & $.39^{* *}\left(.24^{*}\right)$ \\
Play-dates with friends per week & $.24 *(.11)$ & $.20+)$ & $.29 *(.20+)$ \\
Overall rating of child's & $.16(.03)$ & $.03(-.06)$ & $.45^{* *}(.29 * *)$ \\
strengths & & & \\
\hline
\end{tabular}

Measures of Maladjustment

ADHD group

Anxiety (RCMAS)

$\begin{array}{lll}-.08(.01) & -.07(.02) & -.21+(-.13)\end{array}$

Overt Aggression (CSBS)

$.32 *(.21+) \quad .44 *(.25 *) \quad .24 *(.10)$

Relational Aggression (CSBS)

$.31 *(.19)$

$.41 * *(.26 *)$

$.40 * *(.27 *)$

Overall Impairment

$.26 *(.15)$

$.37 *(.22+)$

$.36 *(.21+)$

Control group

$$
\text { Anxiety (RCMAS) }
$$

Overt Aggression (CSBS)

Relational Aggression (CSBS)

\begin{tabular}{lll} 
& & \\
\hline$-.27 *(.13)$ & $-.33 *(.20+)$ & $-.31 *(.17)$ \\
$-.17(-.08)$ & $-.11(-.02)$ & $.15(.04)$ \\
$-.09(.01)$ & $.02(-.04)$ & $-.03(.03)$
\end{tabular}


Positive Illusions in ADHD Girls - 38

Overall Impairment

$.15(.02)$

$.21+(.10)$

$.12(.03)$

Note. RCMAS = Revised Children's Manifest Anxiety Scale; CDS = Children's Depression

Scale; CSBS = Children's Social Behavior Scale; CIRS= Children's Impairment Rating Scale 